\title{
Editorial: IEEE Transactions on Affective Computing-Challenges and Chances
}

\author{
Björn W. Schuller
}

\begin{abstract}
I $\mathrm{N}$ its eight years, the IEEE Transactions on Affective Computing (TAC) has witnessed a time of great opportunities for the field: Artificial Intelligence (AI) and Machine Learning have recently made great progress increasing the distribution and usage of intelligent solutions in the greater public and commercial world. This progress bears many great chances for Affective Computing, as with increasing intelligence of machines, one may increasingly desire according intelligent systems to also possess emotional intelligence as the "next big thing" in commercial exploitation of AI-the Artificial Emotional Intelligence or AEI for short. To give but a few examples, with the advent of spoken language assistants in our homes, and the day-by-day rising usage of such assistants on smart phones and personal computers, it seems more than timely to also lend these assistants the ability to understand their users' emotions and react appropriately to them. Similarly, there is a huge trend in measuring oneself in many ways to track activity, steps, heart rate, sleep time, and whatnots 24/7—one may easily expect emotion tracking to become of broad interest soon, as well, which certainly also bears high promises for serious medical applications. As a last example, with gradually smart retrieval of multimedia, the emotional aspect will likely soon play a much more important role, when-for example-asking your retrieval agent for some funny pictures, bluesy music, or a movie loaded with tension and surprises. Obviously, this also bears a huge challenge for robustness, as the expectancy will be nothing but high once Affective Computing finds its way into the broad consumer market where severe real-world conditions need to be faced. Cross-cultural issues are just one factor to be named which still need many answers on the research's end. Likely, however, such problems will be eased by the ongoing exploitation of big data, efficient intelligent crowd sourcing solutions, and novel deep and further learning techniques. Standardisation may be another factor to find more attention in such days of growing industrial exploitation. The biggest challenge may, however, be on the side of ethical, legal, and social implications-the closer Affective Computing will move to everyday usage by thousands or even millions of users, the more these considerations will come to the fore. For all these named different multi-disciplinary and multi-faceted chances and challenges $T A C$ has kept providing a space for the leading research and ideas in the field. The journal also continued to see great activity by guest editors and excellent submissions at high rates to its special sections. Three were closed in 2016, namely on

Best of Bodynets 2014, guest edited by Giancarlo Fortino (University of Calabria, Italy), and Guang-Zhong Yang (Imperial College London, UK),

Laughter Computing. Towards Machines Able to Deal with Laughter, guest edited by Maurizio Mancini (University of Genoa, Italy), Radoslaw Niewiadomsky (University of Genoa, Italy), Shuji Hashimoto (Waseda University, Japan), Mary Ellen Foster (University of Glasgow, UK), Stefan Scherer (USC, USA), Gualtiero Volpe (University of Genoa, Italy), and

Toward Commercial Applications of Affective Computing, guest edited by Domen Novak (University of Wyoming, USA), Guillaume Chanel (Swiss Center for Affective Sciences, Switzerland), Alexander Koenig (BMW Group, Germany), and Philippe Guillotel (Technicolor Research \& Innovation, France).

Further, two calls were just closed, namely on

Human Behavior Analysis "in-the-wild" guest edited by Stefanos Zafeiriou (Imperial College London, UK), Guoying Zhao (University of Oulu, Finland), Irene Kotsia (International Hellenic University, Greece and Middlesex University, UK), Mihalis Nicolaou (Goldsmiths, University of London, UK), and Jeffrey Cohn (University of Pittsburgh and CMU, USA), and Affective Reasoning for Big Social Data Analysis guest edited by Erik Cambria (Nanyang Technological University, Singapore), Amir Hussain (University of Stirling, UK), and Alessandro Vinciarelli (University of Glasgow, UK).

Finally, one call is currently open, namely for

Artificial Personality Analysis, guest edited by Sergio Escalera (Computer Vision Center and University of Barcelona, Spain), Xavier Baró (Universitat Oberta de Catalunya \& Computer Vision Center, Spain), Isabelle Guyon (INRIA, France), Hugo Jair Escalante (INAOE, Mexico), and Domen Novak (University of Wyoming, USA).

Besides, the editorial board has seen some changes, as 12 long-running members have come up for retirement: I would like to particularly thank Jeremy Bailenson (Stanford University, USA), Rafael Calvo (University of Sydney, Australia), Jeffrey Cohn (University of Pittsburgh, USA), Alan Hanjalic (TU Delft, the Netherlands), Qiang Ji (Rensselaer Polytechnic Institute, USA), Seong-When Lee (Korea University, Korea), Christine Lisetti (Florida International University, USA),
\end{abstract}


Stacy Marsella (Northeastern University, USA), Rada Mihalcea (University of Michigan, USA), Shrikanth Narayanan (USC, USA), Catherine Pelachaud (CNRS, France), and Georgios Yannakakis (University of Malta, Malta) for their wonderful service as Associate Editors over the last years.

In exchange, it is with greatest joy that I am able to announce that again we could win the following highly esteemed seven new members for the editorial board given in alphabetical order as follows:

Florian Metze (CMU, USA), Grace Ngai (Hong Kong Polytechnic University, China), Albert Ali Salah (Bogazici University, Turkey), Friedhelm Schwenker (Uni Ulm), Stefan Steidl (FAU, Germany), Yi-Hsuan Yang (Academica Sinica, Taiwan), and Stefanos Zafeiriou (Imperial College London, UK).

Further, manifold novel reviewers have taken up service for the journal—a very special thank you is dedicated on behalf of the journal to the great effort put into highest quality reviews over the years.

The journal could not exist without the outstanding work of the Steering Committee (SC) members: For the IEEE Computer Society Jonathan Gratch (SC Chair), Arvid Kappas, Hatice Gunes, and Mohammad Soleymani; for the IEEE Systems, Man and Cybernetics Society Goutam Chakraborty, and Weiming Shen; and for the IEEE Computational Intelligence Society Nikhil R. Pal. The same holds for the marvellous support of the staff at IEEE including Samantha Jacobs, Jennifer Carruth, Kathy Santa Maria, Hilda Carman, Kimberly Sperka, and Marisa Peacock.

It remains to note that I am delighted to have been re-elected for the second period as the journal's Editor in Chief. It will be my very joy to dedicate my greatest zeal and ideas to keep this brilliant forum of scientific exchange the leading journal on Affective Computing research for the best authors and readers.

With best wishes for 2017. 